\title{
An Analysis to Validate the Travel Time in Order to Develop an Animated Simulation to Reproduce the Congestion Situation during a Massive Downpour
}

\author{
Mohammad Hannan Mahmud Khan, Motohiro Fujita and Wisinee Wisetjindawat \\ Scientific and Engineering Simulation Department, Nagoya Institute of Technology, Nagoya, Japan
}

\begin{abstract}
It is undesirable to face traffic congestion during an emergency. Congestion does not only cause delay to returning home commuters but also impede emergency vehicles. It is necessary to identify the congestion location as well as congestion severity in order to find the causes and countermeasures, furthermore setting priorities among remedial steps to alleviate the severity. In this study, we prepare an animated simulation of returning home behavior of commuters during a downpour. A taxi probe data was used to prepare the simulation. However, a direct visualization of the raw data does not produce a comprehensive representation of the real traffic situation. This study aims at reproducing efficiently the real traffic situation under such condition that can be easily recognized by a simple and quick glance. This paper discusses the method to reproduce the data as well. The proposed method provides a better realization of the traffic condition. The animated simulation that would be built from a preferable pattern can be used to explore the level of congestion in numerous locations and to evaluate the performance of countermeasures to overcome the congestion.
\end{abstract}

\section{Introduction}

In recent years, coastal cities including Nagoya, Toyota, and Ise have been facing a series of downpour disaster quite frequently. For example, on 20th September 2011 Nagoya city experienced a devastating downpour caused by Typhoon Roke.

Strong winds and driving rain led to the cancellation of train services in Nagoya. Moreover, several companies were forced to close the facilities as a precaution. This situation increased the number of commuters bounded for home. Consequently, they chose to travel by cars as the only available option for returning home. This produced an additional traffic load. Flash floods and extreme river waves covered roads and bridges. It reduced the road capacity and generated a further dimension of congestion severity.

During an emergency, severe congestion is not welcomed since it related to life and death of victims. Therefore, alleviating congestion is one of the greatest challenges in traffic operations. As a result, this study tries to establish a traffic simulation to inspect the performance of countermeasures to alleviate traffic congestion.

Most of the recent studies focus on pre-disaster evacuation and post-disaster network reconstruction [1]. However, quite a few studies worked on the situation during a disaster, which is further subtle on typhoon as a catastrophe. Y. Hiroi, N. Sekiya, R. Nakajima, S.
Waragai, and H. Hanahara [2] conducted a questionnaire survey to study and discuss the usage of smart technology while returning home during an earthquake. T. Kazuyuki, F. Makoto, S. Shigeki, and O. Yusuke [3] developed models to describe the preference for returning home during an earthquake. Though these works investigate on the returning home passengers, yet lack considering downpour situation.

Study on downpour situations, such as M. Fujita, M. Jun, and K. Suzuki [4] focused on individual attitude and awareness of typhoon evacuation among public transport users for any future downpour using a questionnaire survey. However, an analytic approach is absent there even though it is among quite a few studies focusing typhoon.

In addition, M. H. M. Khan, W. Wisetjindawat, M. Fujita, and K. Suzuki [5] investigated the GIS approach using taxi probe data and examined the travel time accuracy. Even it discussed the potential for using probe data over traditional survey data, the development of a validation system was lacking there. Moreover, M. H. M. Khan, W. Wisetjindawat, and M. Fujita [6] analyzed the congestion situation through GIS approach utilizing taxi probe data, yet that was lack of a proper justification.

The previous literatures reveal that no existing analysis focused on the commuters returning home through roadway while no other possible travel mode during a heavy torrential rain event. They also lacked the presence of an animated real-time simulation in this 
context. Our simulation will be helpful in overcoming this lack of a well-developed simulation. It could find applications that include exploration of level of congestion intensities in several locations and also can be used to evaluate the performance of countermeasures.

\section{Outline of taxi probe data}

We used taxi car as probe vehicle in which the data was received from a Taxi Probe Research Center. The vehicle is capable of sending the data of Positioning with time, Speed, Angle and Vehicle status. A good number of GPS device installed cars (taxis) was left moving in a traffic stream.

We have received the recorded data for three consecutive days, 19th to 21st of September 2011 for 72 hours long. The recording interval of recording the data was an average of $200 \mathrm{~m}$ traveled path and 20 s of travel duration. The location for recording is Aichi prefecture with most of the vehicles moving in and around Nagoya city. As our probe vehicle is a taxi car therefore, vehicle status data such as with/without passenger were important concern in the data preparation and analysis as well.

\section{Creating the basic animated simulation during downpour disaster}

In this section, we construct the basic animated simulation using taxi probe data to represent the congestion severity around Nagoya city during downpour disaster.

The purpose of this simulation is to communicate with drivers for a better recognition of the congestion severity during disaster. In general, the number of probe data during disaster is inadequate to represent to real traffic situation. Especially, when the congestion level is worsening, the probe cars cannot enter to the traffic; hence the number of probe data becomes much smaller. During severe traffic congestion, the probe vehicles are also strictly running along with other vehicles in the traffic stream. It is possible to consider that other vehicles exist in between the probe vehicles. This study prepares a visualization of the probe vehicles with a proper size and color for a better representation of the congestion level. In addition, using the probe vehicles cannot represent other vehicles in between the probe cars. Thus, the probe data are expanded to represent the other following vehicles in the traffic stream.

GIS is used to prepare the simulation. In Figure 1, a lot of circles with difference in size and colors are shown. These circles denote probe data for that time window. Time window is the particular period of time, for which length the data are plotted on a single frame. As in the legend, the size and color vary according to the speed classes. The slower vehicles are with larger sizes and the most red in color.

GPS data was recorded as the vehicle changed its position for a particular distance. It resulted in fewer data for the lower speed vehicles. Therefore, representation of unmodified data would obviously underestimate the real situation. For this reason, we require augmenting the lower speed values to the extent of real life data volume.

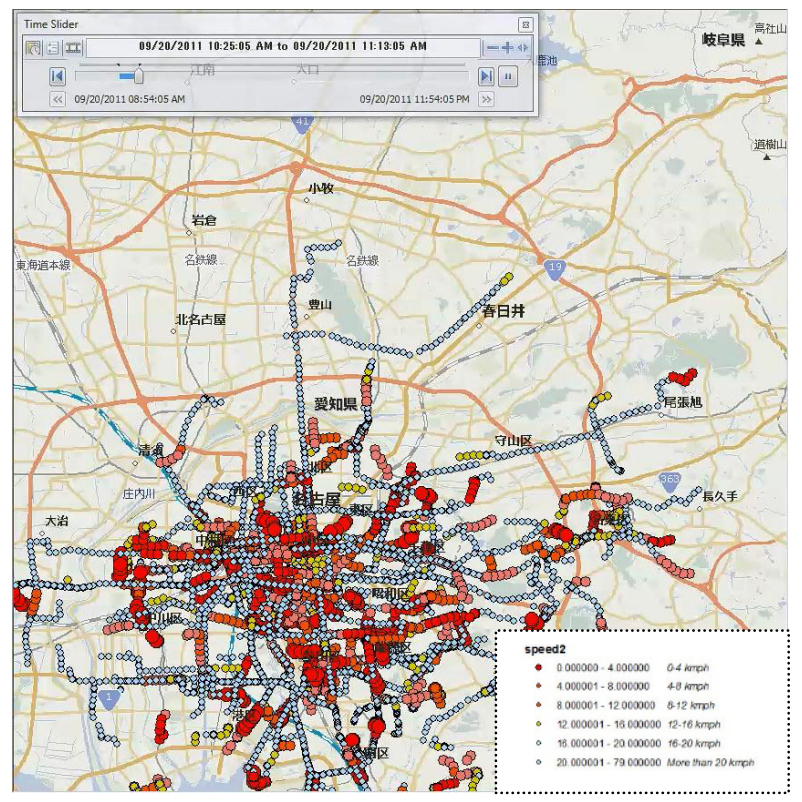

Figure 1. Simulation output window from basic animated simulation.

Table 1. Factors for different speed classes.

\begin{tabular}{|c|c|}
\hline Speed Class & $\begin{array}{c}\text { Multiplication } \\
\text { Factor }\end{array}$ \\
\hline $0-4 \mathrm{~km} / \mathrm{h}$ & 4 \\
\hline $4-8 \mathrm{~km} / \mathrm{h}$ & 4 \\
\hline $8-12 \mathrm{~km} / \mathrm{h}$ & 4 \\
\hline $12-16 \mathrm{~km} / \mathrm{h}$ & 1 \\
\hline
\end{tabular}

We proposed the multiplication factors as in Table 1, for different speed classes. We achieved this factor after experimenting with a number of patterns. We could not validate the output at that time.

Therefore, in this study we would do the evaluation to propose a well suited pattern of multiplication factor for the better portrayal of the actual situation.

\section{Methodology}

\subsection{Data transformation}

The data recorded are simply comprised different location with time-tagged information along with point speed recording. In general, GPS contains huge dataset to track the vehicle down along its route that makes the dataset large in volume. To process the data, we transformed the raw data into desired indicators. We used Java platform to facilitate data transformation.

\subsubsection{Preparation of probe data}

Data reduction is one of the essential parts of the research. As GPS data is the continuous trajectory for a vehicle for the specific duration of recording, therefore, data 
reduction process is introduced to identify OD trips and desired travel indicators. It needs to cut the continuous trajectory into trip ends primarily by vehicle status. The origin of any trip is defined by the change of another vehicle status to occupied status contrary to determine the destination of that trip. The trips without passengers were not included in the data, as there are chances for any unusual driving behavior. In this research, we proposed vehicle status as an indicator to define origin and destination of a trip.

It seems difficult to differentiate between a genuine trip end and just stopping there while a taxi waits at a signalized intersection for a longer duration. As congestion situation is observed in this research, therefore appropriate care is required to handle these data. As long as there is a passenger in the car has an indication of not waiting for any unusual reasons but to follow the travel path. So, long stops for any journey, carrying passengers played a substantial role to determine travel time.

This study concentrated on the return home behavior. For this reason, the trips outbound from the center of Nagoya city is only filtered out from the whole dataset based on their positioning information and approaching direction. We used the probe data as a benchmark dataset to perform the experiment. Probe data consisted of travel information for a number of taxis' wherein every Taxi runs multiple trips. We stored travel time and distance traveled for every change in position for each trip by the difference in the time stamp and by determining linear distance respectively.

\subsubsection{Preparation of simulation data}

In order to mimic the actual situation, we proposed several patterns of data expansion. We factored the number data regarding their speed classes to prepare the simulation environment, including the pattern we chose so far, as in Table 2.

In the earlier study, we proposed to set the time window for 48 minutes therein. We further augment the dataset to make the dataset similar to the simulation presented in the study.

\subsection{Data analysis}

We have analyzed the data for the day of 20th September 2011 as the downpour was the most severe on the same day in Nagoya city. Simulation output showed a continuous trajectory of multiple frames for a particular time window. Therefore, firstly, we need to begin the analysis by filtering out the data of a given time window.
We considered each test probe data as a square object with a particular area. The initial area of that object was set as the size of the largest circle in the simulation, as the largest one would draw the attention while displaying the simulation output. In this study, we examined three different sizes to determine the most suitable one for the development of the new animated simulation. In Table 3, we can observe the pattern development. Firstly, the variation in multiplication factor, from Table 2. Secondly, the variations in setting the initial area of the test probe object.

The object traveled through its path and explore for any simulation speed data inside the boundary based on positioning data. The observer would store the top most data in case of multiple data. Concurrently, the observer varies its area while the speed data is not from the lowest speed class. It modified its area for three instances up until to achieve the lowest speed data. On the contrary, it stored a value of $20 \mathrm{~km} / \mathrm{h}$ as a speed data. This approach is unique, as the probe observer itself was picking up the speed data that is supposed to be displayed.

The concept of changing area of the square observer was very essential. It helped to facilitate lower speed classes by varying the area.

So, the observer recorded the speed value which would be displayed in the simulation. Then, we used the distance data of the test probe object to determine the travel time by the speed distance relationship. Next, the data were grouped by vehicle and particular trips to achieve the overall travel time for any given trip. Finally, both of the travel time, traveled in actual by probe data and data recorded by the Observer probe were compared.

The whole process was repeated several times for patterns from 1A through 5C (Table 3). Then, the performance indices are determined for all of the patterns to propose the most effective pattern for the development of the animated simulation.

Table 2. Pattern distribution for simulation data

\begin{tabular}{|c|c|c|c|c|c|}
\hline $\begin{array}{c}\text { Speed } \\
\text { Class }\end{array}$ & $\begin{array}{c}\text { Pattern } \\
\mathbf{1}\end{array}$ & $\begin{array}{c}\text { Pattern } \\
\mathbf{2}\end{array}$ & $\begin{array}{c}\text { Pattern } \\
\mathbf{3}\end{array}$ & $\begin{array}{c}\text { Pattern } \\
\mathbf{4}\end{array}$ & $\begin{array}{c}\text { Pattern } \\
\mathbf{5}\end{array}$ \\
\hline $\begin{array}{c}0-4 \\
\mathrm{~km} / \mathrm{h}\end{array}$ & 2 & 3 & 4 & 6 & 12 \\
\hline $\begin{array}{c}4-8 \\
\mathrm{~km} / \mathrm{h}\end{array}$ & 2 & 3 & 4 & 5 & 12 \\
\hline $\begin{array}{c}8-12 \\
\mathrm{~km} / \mathrm{h}\end{array}$ & 2 & 2 & 4 & 4 & 8 \\
\hline $\begin{array}{c}12-16 \\
\mathrm{~km} / \mathrm{h}\end{array}$ & 1 & 1 & 1 & 1 & 1 \\
\hline
\end{tabular}

Table 3. Detail distribution of various patterns

\begin{tabular}{|c|c|c|c|c|c|c|c|c|c|c|c|c|c|c|c|c|}
\hline \multirow{3}{*}{$\begin{array}{l}\text { Speed } \\
\text { Class }\end{array}$} & \multirow{3}{*}{$\begin{array}{l}\text { Size of the } \\
\text { Observer } \\
\text { (m) }\end{array}$} & \multicolumn{15}{|c|}{ Patterns } \\
\hline & & $1 A$ & $1 B$ & $1 C$ & $2 A$ & $2 B$ & $2 C$ & $3 A$ & $3 B$ & $3 C$ & $4 A$ & $4 B$ & $4 C$ & $5 A$ & $5 B$ & $5 C$ \\
\hline & & 52 & 78 & 104 & 52 & 78 & 104 & 52 & 78 & 104 & 52 & 78 & 104 & 52 & 78 & 104 \\
\hline \multicolumn{2}{|c|}{$0-4 \mathrm{~km} / \mathrm{h}$} & \multicolumn{3}{|c|}{2} & \multicolumn{3}{|c|}{3} & \multicolumn{3}{|c|}{4} & \multicolumn{3}{|c|}{6} & \multicolumn{3}{|c|}{12} \\
\hline \multicolumn{2}{|c|}{$4-8 \mathrm{~km} / \mathrm{h}$} & \multicolumn{3}{|c|}{2} & \multicolumn{3}{|c|}{3} & \multicolumn{3}{|c|}{4} & \multicolumn{3}{|c|}{5} & \multicolumn{3}{|c|}{12} \\
\hline \multicolumn{2}{|c|}{$8-12 \mathrm{~km} / \mathrm{h}$} & \multicolumn{3}{|c|}{2} & \multicolumn{3}{|c|}{2} & \multicolumn{3}{|c|}{4} & \multicolumn{3}{|c|}{4} & \multicolumn{3}{|c|}{8} \\
\hline \multicolumn{2}{|c|}{$12-16 \mathrm{~km} / \mathrm{h}$} & \multicolumn{3}{|c|}{1} & \multicolumn{3}{|c|}{1} & \multicolumn{3}{|c|}{1} & \multicolumn{3}{|c|}{1} & \multicolumn{3}{|c|}{1} \\
\hline
\end{tabular}




\section{Results and discussions}

We will briefly discuss the statistical output from the analysis for the time window of 1400 hours to 1448 hours. We chose the following graphs to report the outcome of the experiment. Fig. 2 is the output of pattern $1 \mathrm{~A}$ as described in Table 3.

We observe in Figure 2 that most of the trip time data are close to each other. Trip time data is the travel time required for each trip for the probe and simulation data as well. Figure 3 displays the output distribution of pattern 1C. The trip time data are quite confined, which provided a good fit line. It also seems that the simulation trip time is quite similar to the probe output.

The root mean square error (RMSE) defined by equations (1) is used to judge the proficiency:

$$
R M S E=\sqrt{\frac{1}{N} \sum_{i=1}^{N}\left(x_{i}-\hat{x}_{i}\right)^{2}}
$$

where, $\mathrm{N}$ is the total number of testing cases, $\hat{x}_{i}$ the predicted simulation trip time and $x_{i}$ the probe trip time.

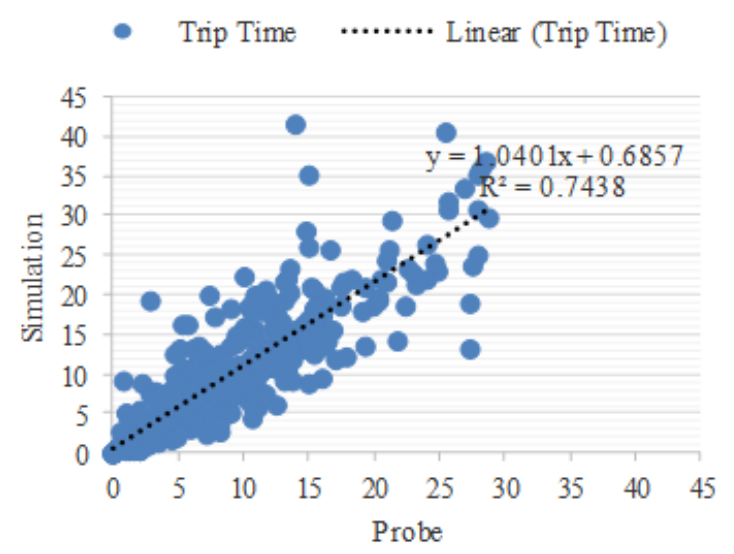

Figure 2. Travel time for different trips (Minutes)

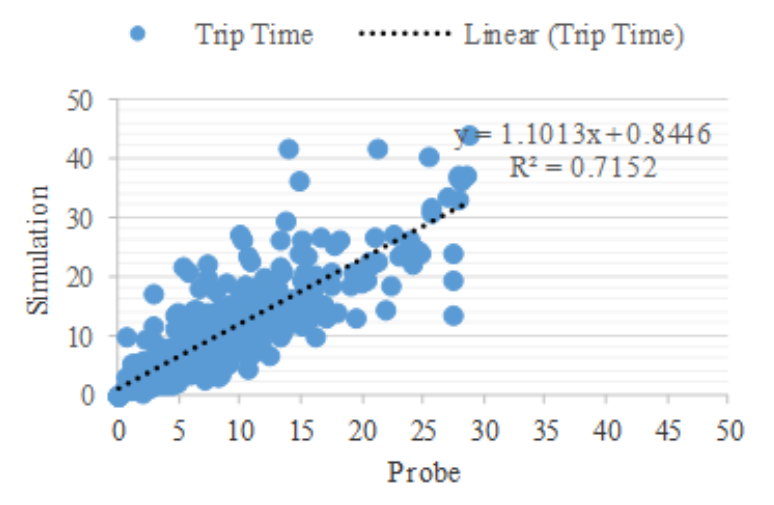

Figure 3. Travel time for different trips (Minutes)

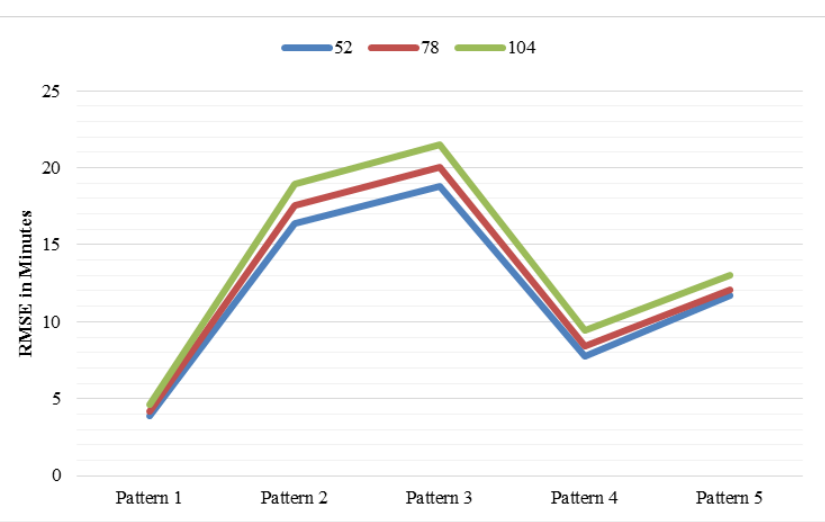

Figure 4. Variations of RMSE (Minutes)

In Fig. 4, the RMSE values are drawn for three different sizes of the probe observer. According to the RMSE value, we find the prediction for Pattern 1 and Pattern 4 is better than any other patterns. We can conclude that, pattern $1 \mathrm{~A}$ as in Table 3 can be the most preferable pattern to develop the simulation.

\section{Conclusion}

A well representative animated simulation is essential to communicate with drivers for a comprehensive perception of the congestion severity during a disaster. With this animated simulation, the congested locations can be efficiently recognized in order to anticipate proper countermeasures to mitigate the congestion severity. However, the available probe data was insufficient to represent the real situation. Therefore, the probe data should be expanded to the extent of the real traffic volume for a better portrayal of actual situation. This study has simulated the returning home commuters in different patterns and compared the travel time with the actual movement of the probe vehicle during the downpour.

From the results, pattern $1 \mathrm{~A}$ is the most preferable pattern to prepare the simulation. From the simulation we can perceive that, the central Nagoya was heavily congested and the roads with bridges and tunnels were congested as well. This simulation will work as a tool to communicate with the drivers for a comprehensive understanding of the traffic situation for any particular time. However, this paper could not include the consequences on the driver's decision to depart for the journey. This study still require a proper justification for model calibration and validation, which would be considered in the future work. As a transferability of this work, the prepared simulation can be demonstrated to the drivers in order to collect their opinions and these opinions can be used for a further analysis on departure choice. Our future work will also focus on how to use this simulation for forecasting the travel time for commuters simply by adjusting the severity of downpour.

\section{References}

1. H. Yusuke, K. Masao, Traffic Monitoring immediately after a major natural disaster as 
revealed by probe data - A case in Ishinomaki after the Great East Japan Earthquake, Tr. Res. Part A: Pol. And Prac., 75, 1-15 (2015)

2. Y. Hiroi, N. Sekiya, R. Nakajima, S. Waragai, H. Hanahara, Questionnaire survey concerning stranded commuters in metropolitan area in the East Japan Great Earthquake, J. Ins. Soc. Saf. Sc., 15, 343-353 (2011)

3. T. Kazuyuki, F. Makoto, S. Shigeki, O. Yusuke, Behavior analysis of the people having difficulty in going home in Tokyo metropolitan area-the 2011 off the pacific coast of Tohoku Earthquake, Proc. of $15^{\text {th }}$ World Conference on Earthquake Engg. (2012)
4. M. Fujita, M. Jun, K. Suzuki, Behavioral analysis of homebound public transport users during downpour conditions, J. of the As. Tr. St., 1(3), 318-333 (2011)

5. M. H. M. Khan, W. Wisetjindawat, M. Fujita, K. Suzuki, An analysis of Probe data on traffic congestion during the Typhoon using GIS application, Proc. of the Est. As. Soc. for Tr. St. 9 (2013)

6. M. H. M. Khan, W. Wisetjindawat, M. Fujita, Analysis of traffic congestion due to Typhoon using taxi probe data, Proceedings of JSCE, 47 (2013) 